\title{
ON THE MEAN ERGODIC THEOREM OF SINE
}

\author{
STUART P. LLOYD
}

\begin{abstract}
Robert Sine has shown that $(1 / n)\left(I+T+\cdots+T^{n-1}\right)$, the ergodic averages, converge in the strong operator topology iff the invariant vectors of $T$ separate the invariant vectors of the adjoint operator $T^{*}, T$ being any Banach space contraction. We prove a generalization in which (spectral radius of $T$ ) $\leqq 1$ replaces $\|T\| \leqq 1$, and any bounded averaging sequence converging uniformly to invariance replaces the ergodic averages; it is necessary to assume that such sequences exist.
\end{abstract}

1. Introduction. Let $T: \mathcal{X} \rightarrow \mathscr{X}$ be a bounded linear operator of spectral radius $r(T) \leqq 1$ on real or complex Banach space $\mathcal{X}$, let $\mathfrak{N}=\{x \in \mathfrak{X}: T x$ $=x\}$ be the invariant vectors of $T$, and let $\Re=\left\{\xi \in X^{*}: T^{*} \xi=\xi\right\}$ be the invariant vectors of the adjoint operator $T^{*}: \mathscr{X}^{*} \rightarrow \mathscr{X}^{*}$. Robert Sine has shown [4] that in the case $\|T\| \leqq 1$, the condition $\langle\mathscr{T}$ separates $\mathscr{\Re}$ 〉 is necessary and sufficient for strong convergence of the ergodic averages $(1 / n)\left(I+T+\cdots+T^{n-1}\right) x, x \in \mathcal{X}$. We show here that $\langle\mathscr{T}$ separates $\mathfrak{R}\rangle$ is necessary and sufficient for strong convergence of any bounded regular invariant summability method, and that the limiting operator, a projection onto $\mathfrak{T}$, is independent of the method when it exists.

2. Mean ergodic theorem. Let $\mathcal{Q}_{1}$ be the set of power series $p(z)=\sum_{i=0}^{\infty} p_{i} z^{i}$ which have radius of convergence greater than 1 and are such that $\sum_{i=0}^{\infty} p_{i}$ $=1$. With $\mathscr{B}(\mathscr{X})$ the bounded linear operators on $\mathscr{X}$, each $p \in \mathbb{Q}_{1}$ determines $p(T)=\sum_{i=0}^{\infty} p_{i} T^{i} \in \mathscr{B}(\mathcal{X})$, the series converging in the uniform operator topology. We put $\mathscr{P}_{1}=\left\{p(T): p \in \mathbb{Q}_{1}\right\}$, noting that if $P \in \mathscr{P}_{1}$ then $P x=x$, $x \in \Re$.

We introduce further $\mathscr{P}_{1}(M)=\left\{P \in \mathscr{P}_{1}:\|P\| \leqq M\right\}$ for $1 \leqq M<\infty$, and also $\mathscr{P}_{1}(M, \varepsilon)=\left\{P \in \mathscr{P}_{1}(M):\|(I-T) P\| \leqq \varepsilon\right\}, 1 \leqq M<\infty, \varepsilon>0$. Our basic assumption will be:

(UI) There exists $1 \leqq M_{0}<\infty$ such that

$$
\mathscr{P}_{1}\left(M_{0}, \varepsilon\right) \neq \varnothing \text { for each } \varepsilon>0 \text {. }
$$

In the terminology of Day [1], this is the assertion that $\mathscr{P}_{1}$ contains bounded sequences $\left\{P_{n}\right\}$ converging uniformly to invariance, i.e., $\lim _{n}\left\|P_{n}-T P_{n}\right\|=0$. In $\$ 3$ we give various sufficient conditions for (UI).

Let $\overline{\mathcal{P}}_{1}$ be the closure of $\mathscr{P}_{1} \subset \mathscr{B}(\mathcal{X})$ in the strong operator topology (SOT), and let $\overline{\mathscr{S}}_{1}(M, \varepsilon)$ be the SOT closure of $\mathscr{P}_{1}(M, \varepsilon)$.

THEOREM 1. If (UI) holds then (i) is equivalent to (ii):

Presented to the Society, January 26, 1975; received by the editors March 1, 1975.

AMS (MOS) subject classifications (1970). Primary 47A35.

Key words and phrases. Mean ergodic theorem. 
(1) (i) $\Re$ separates $\Re$;

(ii) There exists a projection $Q \in \overline{\mathcal{P}}_{1}$ onto $\mathfrak{R}$, necessarily unique, and $\bigcap_{\varepsilon>0} \bar{P}_{1}(M, \varepsilon)=\{Q\}$ for any $M \geqq M_{0}$.

Proof. We show first that $\overline{\mathscr{P}}_{1}$ contains at most one projection $Q$ onto $\mathfrak{T}$. Suppose $\lim _{\nu} P_{\nu}=Q$ in SOT with $\left\{P_{\nu}\right\} \subset \mathscr{P}_{1}$ a generalized sequence and $Q$ a projection onto Th. Such a projection commutes with $T$, from

$$
Q=T Q=T \lim _{\nu} P_{\nu}=\lim _{\nu} T P_{\nu}=\lim _{\nu} P_{\nu} T=Q T,
$$

the limits being SOT. If $\lim _{\nu} P_{\nu}^{\prime}=Q^{\prime}$ and $\lim _{\nu} P_{\nu}^{\prime \prime}=Q^{\prime \prime}$, then $Q^{\prime}=Q^{\prime} P_{\nu}^{\prime \prime}$ $=Q^{\prime} Q^{\prime \prime}=Q^{\prime \prime}$, whence $Q$ is unique in $\overline{\mathcal{P}}_{1}$ if it exists.

(ii $\Rightarrow$ i) Let us prove that if projection $Q$ exists then the adjoint projection $Q^{*}: \mathscr{X}^{*} \rightarrow X^{*}$ is onto $\Re$. From $Q=T Q=Q T$ follows $Q^{*}=Q^{*} T^{*}$ $=T^{*} Q^{*}$, so that $Q^{*}$ has range in $\Re$. If $Q=\lim _{\nu} P_{\nu}$ with $\left\{P_{\nu}\right\} \subset \mathscr{P}_{1}$ and if $x \in \mathcal{X}, \xi \in \mathcal{X}$, then

$$
(x, \xi)=\left(x, P_{\nu}^{*} \xi\right)=\left(P_{\nu} x, \xi\right)=(Q x, \xi)=\left(x, Q^{*} \xi\right),
$$

giving $Q^{*} \xi=\xi, \xi \in \Re$, whence $Q^{*}$ is onto $\Re$.

Suppose $0 \neq \xi \in \Re$, and let $x \in \mathcal{X}$ be such that $(x, \xi) \neq 0$. Then $(Q x, \xi)$ $=\left(x, Q^{*} \xi\right)=(x, \xi) \neq 0$, so that $Q x \in \mathfrak{N}$ separates $\xi$ and 0 . That is, $\mathscr{T}$ separates $\%$.

( $\mathrm{i} \Rightarrow$ ii) Let functional $\Phi(x), x \in \mathcal{X}$, be defined by

$$
\Phi(x)=\lim _{\varepsilon \downarrow 0} \sup _{P^{\prime}, P^{\prime \prime}}\left\{\left\|P^{\prime} x-P^{\prime \prime} x\right\|: P^{\prime}, P^{\prime \prime} \in \mathscr{P}_{1}(M, \varepsilon)\right\}, \quad x \in \mathcal{X},
$$

for any fixed $M \geqq M_{0}$. The properties

(a) $\Phi(x+y) \leqq \Phi(x)+\Phi(y), x, y \in \mathfrak{X}$,

(b) $\Phi(c x)=|c| \Phi(x), x \in \mathcal{X}$, scalar $c$,

(c) $0 \leqq \Phi(x) \leqq 2 M\|x\|, x \in \mathcal{X}$,

(d) $\Phi(x)=0, x \in \Re$,

(e) $\Phi(x-T x)=0, x \in \mathfrak{X}$,

are straightforward or obvious; e.g. for (e) we use

$$
\begin{aligned}
\left\|P^{\prime}(x-T x)-P^{\prime \prime}(x-T x)\right\| & \leqq\left[\left\|(I-T) P^{\prime}\right\|+\left\|(I-T) P^{\prime \prime}\right\|\right]\|x\| \\
& \leqq 2 \varepsilon\|x\|, \quad x \in \mathcal{X} \text { and } P^{\prime}, P^{\prime \prime} \in \mathscr{P}_{1}(M, \varepsilon) .
\end{aligned}
$$

Let $\Phi^{0}$ denote the set of all $\xi \in \mathcal{X}^{*}$ satisfying $(x, \xi) \leqq \Phi(x), x \in \mathcal{X}$; by the Hahn-Banach theorem, $\Phi(x)=\max _{\xi}\left\{(x, \xi): \xi \in \Phi^{0}\right\}, x \in \mathcal{X}$. Properties (d), (e) of $\Phi$ yield properties: $\left(d^{*}\right) \xi \in \mathscr{N}^{\perp} ;\left(\mathrm{e}^{*}\right) \xi \in \mathfrak{N}$, for the $\xi \in \Phi^{0}$, where $\Re^{\perp} \subset \mathscr{X}^{*}$ is the annihilator of $\Re$. Now, condition (1)(i) is the assertion $\Re^{\perp} \cap \Re=0$; from $\Phi^{0} \subset \Re^{\perp} \cap \Re$ just shown follows then $\Phi^{0}=0$ and hence $\Phi=0$.

Let $\left\{\eta_{n}\right\}$ be any sequence of positive numbers such that $\lim _{n} \eta_{n}=0$, let $P_{n}$ for each $n$ be an arbitrarily chosen member of $\mathscr{P}_{1}\left(M, \eta_{n}\right)$, and put $\varepsilon_{n}$ $=\max _{r \geqq n} \eta_{r}$. Any such sequence $\left\{P_{n}\right\}$ is SOT Cauchy when $\Phi=0$, from 


$$
\begin{aligned}
\lim _{n \rightarrow \infty} \sup _{n \leqq r<s}\left\|P_{r} x-P_{s} x\right\| \\
\quad \leqq \lim _{n \rightarrow \infty} \sup _{P^{\prime}, P^{\prime \prime}}\left\{\left\|P^{\prime} x-P^{\prime \prime} x\right\|: P^{\prime}, P^{\prime \prime} \in \mathscr{P}_{1}\left(M, \varepsilon_{n}\right)\right\} \\
\quad=\lim _{\varepsilon \downarrow 0} \sup _{P^{\prime}, P^{\prime \prime}}\left\{\left\|P^{\prime} x-P^{\prime \prime} x\right\|: P^{\prime}, P^{\prime \prime} \in \mathscr{P}_{1}(M, \varepsilon)\right\} \\
\quad=\Phi(x)=0, \quad x \in \mathscr{X} ;
\end{aligned}
$$

we have used the nested property of the $\left\{\mathscr{P}_{1}(M, \varepsilon): \varepsilon>0\right\}$. The SOT limit $Q=\lim _{n} P_{n}$ is the projection sought.

We remark that when $\Re=0$ the result takes the form: given (UI), $0 \in \overline{\mathscr{P}}_{1}$ iff $\Re=0$.

3. Condition (UI). Consider the familiar ergodic averages $A_{n}$ $=(1 / n) \sum_{i=0}^{n-1} T^{i}, n \geqq 1$, for which $(I-T) A_{n}=\left(I-T^{n}\right) / n$. If these satisfy $\left\|A_{n}\right\| \leqq M_{0}<\infty, n \geqq 1$, and if $\lim _{\inf _{n}}\left\|T^{n}\right\| / n=0$, then (UI) holds, clearly. A fortiori, (UI) holds when $\left\|T^{n}\right\| \leqq M_{0}<\infty, n \geqq 1$; this case is covered by the arguments of [4], although Sine assumes $\|T\| \leqq 1$.

The resolvent of $T$ is given by $R_{\lambda}=\sum_{i=0}^{\infty} T^{i} / \lambda^{i+1}$ when $|\lambda|>1 \geqq r(T)$, the series converging in the uniform operator topology. If we introduce $P_{\lambda}=(\lambda-1) R_{\lambda}$ for $|\lambda|>1$, then $P_{\lambda} \in \mathscr{P}_{1}$, and it is easily verified that $(I-T) P_{\lambda}=(\lambda-1)\left(I-P_{\lambda}\right)$. Thus if the $\left\{P_{\lambda}\right\}$ satisfy

$$
\lim _{\lambda \rightarrow 1 ;|\lambda|>1} \inf _{\lambda}\left\|P_{\lambda}\right\|<M_{0}<\infty
$$

then (UI) holds for such $M_{0}$.

In the other direction, suppose $T$ has index $1<\mu<\infty$ at $\lambda=1$. (The index of $T$ at $\lambda=1$ is the least integer $\mu \geqq 0$ with the property: all vectors $x \in \mathcal{X}$ satisfying $(I-T)^{\mu+1} x=0$ satisfy also $(I-T)^{\mu} x=0[2$, p. 556].)

THeorem 2. If $T$ has index $1<\mu<\infty$ at $\lambda=1$ then (UI) cannot hold and $\overline{\mathcal{P}}_{1}$ contains no projection onto $\Re$.

Proof. If a generalized sequence $\left\{P_{\nu}\right\} \subset \mathscr{P}_{1}$ is SOT convergent to a projection $Q \in \overline{\mathscr{T}}_{1}$ onto $\Re$, then, necessarily,

$$
\lim _{\nu}\left\|(I-T) P_{\nu} x\right\|=\|(I-T) Q x\|=0, \quad x \in \mathfrak{X} .
$$

If $T$ has index $1<\mu<\infty$ at $\lambda=1$, then unit vectors $x_{1}, x_{2} \in \mathcal{X}$ exist such that $T x_{1}=x_{1}, T x_{2}=x_{2}+c x_{1}$ for some $c>0$. From

$$
(I-T) T^{i} x_{2}=T^{i}(I-T) x_{2}=T_{i}\left(-c x_{1}\right)=-c x_{1}, \quad i \geqq 0,
$$

follows $(I-T) P x_{2}=-c x_{1}, P \in \mathscr{P}_{1}$. We have then $\left\|(I-T) P x_{2}\right\|=c>0$, $P \in \mathscr{P}_{1}$, showing that no $Q$ exists, and $\|(I-T) P\| \geqq c>0, P \in \mathscr{P}_{1}$, showing that (UI) fails.

For the same $x_{1}, x_{2}$ an easy calculation gives $P_{\lambda} x_{2}=x_{2}+c x_{1} /(\lambda-1)$, whence

$$
\begin{aligned}
\left\|P_{\lambda}\right\| & \geqq|c /| \lambda-1|-1|, & & |\lambda|>1, \\
& =O(1 /|\lambda-1|), & & |\lambda|>1 \quad \text { and } \lambda \rightarrow 1,
\end{aligned}
$$


when $T$ has index $1<\mu<\infty$ at $\lambda=1$. Thus if some condition on $\left\|P_{\lambda}\right\|,|\lambda|$ $>1, \lambda \rightarrow 1$, is necessary and sufficient for (UI) then it lies between (2) and

$$
\left\|P_{\lambda}\right\|=o(1 /|\lambda-1|), \quad|\lambda|>1, \lambda \rightarrow 1 .
$$

Apart from changes in variable, the following result is the $(C, \alpha)$ generalization of Theorem $1^{\prime}$ of [3]. For $\alpha \neq-1,-2, \ldots$ the $(C, \alpha)$ averages $A_{n}^{(\alpha)}(T)$ of $\left\{T^{n}\right\}$ have as generating function

$$
\left(1-\frac{1}{\lambda}\right)^{-\alpha} R_{\lambda}(T)=\sum_{n=0}^{\infty} \frac{\alpha+n !}{\alpha ! n !} \frac{A_{n}^{(\alpha)}(T)}{\lambda^{n+1}} ; \quad|\lambda|>1,
$$

so that

$$
A_{n}^{(\alpha)}(T)=\frac{\alpha ! n !}{\alpha+n !} \frac{1}{2 \pi i} \int \lambda^{n}\left(1-\frac{1}{\lambda}\right)^{-\alpha R_{\lambda}}(T) d \lambda, \quad n \geqq 0,
$$

the contour being a large circle around the origin, say.

For $\lambda$ in the resolvent set of $T$ let $T_{\lambda} \in \mathscr{B}(\mathcal{X})$ be defined by

$$
T_{\lambda}=P_{\lambda} T=(\lambda-1)(\lambda I-T)^{-1} T=\lambda P_{\lambda}-(\lambda-1) I .
$$

The spectrum of $T_{\lambda}$ is $\sigma\left(T_{\lambda}\right)=\left\{(\lambda-1) \lambda^{\prime} /\left(\lambda-\lambda^{\prime}\right): \lambda^{\prime} \in \sigma(T)\right\}$, and we find $1-\lambda \notin \sigma\left(T_{\lambda}\right)$ provided $\lambda \neq 0,1$. Thus the inversion formula

$$
T=\lambda T_{\lambda}\left[(\lambda-1) I+T_{\lambda}\right]^{-1}
$$

is valid for $\lambda \neq 0,1$ in the resolvent set of $T$; moreover, $r\left(T_{\lambda} /(1-\lambda)\right)<1$ if $|\lambda|>2$. Note that the relations between $T_{\lambda}$ and $T$ admit the involution $T \leftrightarrow T_{\lambda}, \lambda \leftrightarrow 1-\lambda$.

TheOREM 3. For any fixed $\alpha \geqq 0,1 \leqq M<\infty$, the conditions

$$
\text { (i) }\left\|A_{n}^{(\alpha)}(T)\right\| \leqq M, \quad n \geqq 0,
$$$$
\text { (ii) }\left\|A_{n}^{(\alpha)}\left(T_{\lambda}\right)\right\| \leqq M\left|\frac{\lambda-1}{|\lambda|-1}\right|^{\alpha+n}, \quad n \geqq 0,|\lambda|>1 \text {, }
$$

are equivalent. When they are satisfied, (UI) holds and

$$
\left\|P_{\lambda}(T)\right\| \leqq M|(\lambda-1) /(|\lambda|-1)|^{\alpha+1}, \quad|\lambda|>1 .
$$

Proof. In

$$
A_{n}^{(\alpha)}\left(T_{\lambda}\right)=\frac{\alpha ! n !}{\alpha+n !} \frac{1}{2 \pi i} \int \mu^{n}\left(1-\frac{1}{\mu}\right)^{-\alpha} R_{\mu}\left(T_{\lambda}\right) d \mu, \quad n \geqq 0,
$$

we have

$$
R_{\mu}\left(T_{\lambda}\right)=\frac{1}{\mu I-T_{\lambda}}=\frac{1}{\lambda+\mu-1}\left\{I+\frac{\lambda(\lambda-1)}{\lambda \mu I-(\lambda+\mu-1) T}\right\}
$$

the change of variable $\xi=\lambda \mu /(\lambda+\mu-1)$ gives 


$$
\begin{aligned}
& A_{n}^{(\alpha)}\left(T_{\lambda}\right) \\
& =\frac{\alpha ! n !}{\alpha+n !}\left(1-\frac{1}{\lambda}\right)^{\alpha+n} \frac{1}{2 \pi i} \int\left[\frac{\xi}{1-(\xi / \lambda)}\right]^{n}\left(1-\frac{1}{\xi}\right)^{-\alpha}\left[\frac{I}{\xi-\lambda}+R_{\xi}(T)\right] d \xi \\
& =\left(1-\frac{1}{\lambda}\right)^{\alpha+n} \sum_{j=0}^{\infty} \frac{\alpha+n-1+j !}{\alpha+n-1 ! j ! \lambda^{j}}\left\{\frac{\alpha j I}{(\alpha+n)(j+n)}\right. \\
& \left.+\left[1-\frac{\alpha j}{(\alpha+n)(j+n)}\right] A_{n+j}^{(\alpha)}(T)\right\}, \\
& n \geqq 0,|\lambda|>1,
\end{aligned}
$$

with $1<|\xi|<|\lambda|$ on the contour. If $\alpha \geqq 0$ then $0 \leqq \alpha /(\alpha+n) \cdot j /(j+n)$ $\leqq 1$ in the last expression, so if (3)(i) holds, then

$$
\begin{aligned}
\left\|A_{n}^{(\alpha)}\left(T_{\lambda}\right)\right\| & \leqq\left|1-\frac{1}{\lambda}\right|^{\alpha+n}\left(1-\frac{1}{|\lambda|}\right)^{-\alpha-n} M \\
& =\left|\frac{\lambda-1}{|\lambda|-1}\right|^{\alpha+n} M, \quad|\lambda|>1,
\end{aligned}
$$

which is (3)(ii).

In the same way, using the bound (3)(ii) in the inversion formula

$$
\begin{aligned}
& A_{n}^{(\alpha)}(T)=\left(1-\frac{1}{\lambda}\right)^{-\alpha-n} \\
& \cdot \sum_{j=0}^{\infty} \frac{\alpha+n-1+j !}{\alpha+n-1 ! j !(1-\lambda)^{j}}\left\{\frac{\alpha j I}{(\alpha+n)(j+n)}\right. \\
&\left.+\left[1-\frac{\alpha j}{(\alpha+n)(j+n)}\right] A_{n+j}^{(\alpha)}\left(T_{\lambda}\right)\right\}, \\
& n \geqq 0,|\lambda|>2,
\end{aligned}
$$

gives

$$
\left\|A_{n}^{(\alpha)}(T)\right\| \leqq[|\lambda| /(|\lambda|-2)]^{\alpha+n} M, \quad n \geqq 0,|\lambda|>2 ;
$$

we let $|\lambda| \rightarrow \infty$ to obtain (3)(i).

If conditions (3) are satisfied then

$$
\left\|R_{\lambda}(T)\right\| \leqq\left[\frac{|\lambda-1|}{|\lambda|}\right]^{\alpha}\left[1-\frac{1}{|\lambda|}\right]^{-\alpha-1} \frac{M}{|\lambda|}=\frac{|\lambda-1|^{\alpha} M}{(|\lambda|-1)^{\alpha+1}}, \quad|\lambda|>1,
$$

which is (4). For a (UI) sequence we may take $\left\{P_{\lambda_{n}}\right\}$ for some $\left\{\lambda_{n} \downarrow 1\right\}$, or $\left\{A_{n}^{(\alpha+1)}(T)\right\}$, since

$$
\begin{aligned}
A_{n}^{(\alpha+1)}(T) & =\sum_{j=0}^{n} \frac{\alpha+j !}{\alpha ! j !} A_{j}^{(\alpha)}(T) / \sum_{j=0}^{n} \frac{\alpha+j !}{\alpha ! j !}, \\
(I-T) A_{n}^{(\alpha+1)}(T) & =(\alpha+1) /(n+1)\left[I-A_{n+1}^{(\alpha)}(T)\right], \quad n \geqq 0 .
\end{aligned}
$$


Note the slight strenghtening of the result of the first paragraph: if $\left\|A_{n}^{(1)}(T)\right\|$ $\leqq M<\infty, n \geqq 0$, then (UI) holds, with no condition on $\left\|T^{n}\right\| / n$. All that is involved in Theorem 3 is $\left\|T-T_{\lambda}\right\|=O(1 /|\lambda|)$ at $|\lambda| \rightarrow \infty$, of course; the interesting part at $\lambda \rightarrow 1$ has no force.

4. The adjoint projections. Recall that a generalized sequence $\left\{\Gamma_{\nu}\right\} \subset \mathscr{B}\left(\mathscr{X}^{*}\right)$ is $\mathrm{W}^{*} \mathrm{OT}$ convergent to $\Gamma \in \mathscr{B}\left(\mathscr{X}^{*}\right)$ iff $\lim _{\nu}\left(x, \Gamma_{\nu} \xi\right)=(x, \Gamma \xi)$ for each $x$ $\in \mathcal{X}, \xi \in \mathcal{X}^{*}$; and further, that bounded $\mathrm{W}^{*}$ OT closed sets are $\mathrm{W}^{*} \mathrm{OT}$ compact. Let $\mathscr{P}_{1}^{*}(M, \varepsilon) \subset \mathfrak{B}_{\left(\mathcal{X}^{*}\right)}$ be the set of adjoints of members of $\mathscr{P}_{1}(M, \varepsilon)$, and let $\overline{\mathcal{P}}_{1}^{*}(M, \varepsilon)$ denote the $\mathrm{W}^{*}$ OT closure of $\mathscr{P}_{1}^{*}(M, \varepsilon)$. If (UI) holds and $M \geqq M_{0}$, then $\left\{\overline{\mathscr{P}}_{1}^{*}(M, \varepsilon): \varepsilon>0\right\}$ is a nested family of nonempty convex $\mathrm{W}^{*}$ OT compact sets; the intersection $\delta(M)=\cap_{\varepsilon>0} \overline{\mathscr{\Phi}}_{1}^{*}(M, \varepsilon)$, necessarily nonempty, consists of projections onto $\Re$ (with $\delta(M)=\{0\}$ if $\Re=0$ ). The members of $\delta(M)$ commute with $T^{*}$ and satisfy $\Gamma^{\prime} \Gamma^{\prime \prime}=\Gamma^{\prime \prime}, \Gamma^{\prime}, \Gamma^{\prime \prime} \in \delta(M)$, clearly. If the projection $Q \in \bar{\Phi}_{1}$ onto $\Re$ exists, then it satisfies $Q^{*} \Gamma=Q^{*}$, $\Gamma \in \delta(M)$. For, suppose $\lim _{\nu} P_{\nu}^{*}=\Gamma \in \delta(M)$ in W* OT; then

$$
\begin{aligned}
\left(x, Q^{*} \Gamma \xi\right) & =(Q x, \Gamma \xi)=\lim _{\nu}\left(Q x, P_{\nu}^{*} \xi\right)=\lim _{\nu}\left(P_{\nu} Q x, \xi\right) \\
& =(Q x, \xi)=\left(x, Q^{*} \xi\right), \quad x \in \mathcal{X}, \xi \in X^{*} .
\end{aligned}
$$

This and $Q^{*} \Gamma=\Gamma$ give $\Gamma=Q^{*}$, which is to say, $\delta(M)$ is the singleton $\delta(M)=\left\{Q^{*}\right\}$ when $Q$ exists. The author is not able to prove the following plausible converse: If $\delta(M)=\{\Gamma\}$ is a singleton, then $\Gamma=Q^{*}$ for $Q \in \overline{\mathcal{P}}_{1}$ a projection onto $\pi$.

\section{REFERENCES}

1. Mahlon M. Day, Amenable semigroups, Illinois J. Math. 1 (1957), 509-544. MR 19, 1067.

2. Nelson Dunford and Jacob T. Schwartz, Linear operators. I: General theory, Pure and Appl. Math., vol. 7, Interscience, New York, 1958. MR 22 \#8302.

3. A. G. Gibson, A discrete Hille-Yosida-Phillips theorem, J. Math. Anal. Appl. 39 (1972), 761-770. MR $47 \# 881$.

4. Robert C. Sine, A mean ergodic theorem, Proc. Amer. Math. Soc. 24 (1970), 438-439. MR 40 \# 5825 .

Bell Laboratories, Murray Hill, New Jersey 07974 